\title{
Trace compounds confined in SAPO-34 and probable evolution route of coke in MTO process
}

Mingjian Luo*, Bing Hu, Guoliang Mao, Baohui Wang

Provincial Key Laboratory of Oil \& Gas Chemical Technology, College of Chemistry \& Chemical Engineering, Northeast Petroleum University, Daqing 163318, Heilongjiang, P.R. China. 


\section{Experiments}

Properties of SAPO-34 and experiments details were described in our previous works. ${ }^{1,2}$ Reactions were performed at atmosphere pressure and $425^{\circ} \mathrm{C}$ with methanol $\left(\mathrm{WHSV}=1 \mathrm{~h}^{-1}\right)$ or olefin $\left(\mathrm{WHSV}=0.5 \mathrm{~h}^{-1}\right)$ feeds for $200 \mathrm{~min}$. Coke weights were 192.5, 79.7, 70.8 and $6.2 \mathrm{mg}_{\text {coke }} / \mathrm{g}_{\text {cat }}$ for methanol, 1-butene, propene, and ethene feeds, respectively. $15 \mathrm{mg}$ coked SAPO-34 was dissolved by $\mathrm{HF}(0.5 \mathrm{~mL}, 10 \%)$, extracted with $\mathrm{CCl}_{4}(150 \mu \mathrm{L})$, and then $\mathrm{CCl}_{4}$ phase was analyzed on a Thermo Fisher Trace GC-MS equipped with a TR-1 MS capillary column $(30 \mathrm{~m} \times 0.25 \mathrm{~mm} \times 0.25 \mu \mathrm{m})$. The carrier gas was high pure He (99.999\%), constant flow mode, with column flow of $0.5 \mathrm{~mL} / \mathrm{min}$ and split ratio of 20 . The GC condition was $50{ }^{\circ} \mathrm{C}$ for $5 \mathrm{~min}$ (methanol sample: $60^{\circ} \mathrm{C}$ for $2 \mathrm{~min}$ ), ramped to $250^{\circ} \mathrm{C}$ with a rate $5^{\circ} \mathrm{C} / \mathrm{min}$, and kept for $5 \mathrm{~min}$. Mass signal were collected in $\mathrm{m} / \mathrm{z}$ of $15-500$ range.

The identification of confined compounds was mainly based on the matching results of fragment ions spectra with NIST MS database. However, due to the presence of isomers, overlapping of peaks, noise of signal and the low content, and limitation of NIST database, some peaks can't be well matched. Structures of these peaks were deduced with consideration of feed and reaction characteristics. 
Table S1. Identified structures in soluble coke of 1-butene, propene, ethene and methanol.

\begin{tabular}{|c|c|c|c|c|c|c|c|c|}
\hline \multirow[b]{2}{*}{ No. } & \multirow[b]{2}{*}{$\begin{array}{l}\mathrm{Rt}^{\mathrm{a}} \\
\mathrm{min}\end{array}$} & \multirow[b]{2}{*}{$\mathrm{MW}^{\mathrm{b}}$} & \multirow[b]{2}{*}{$\mathrm{MF}^{\mathrm{c}}$} & \multirow[b]{2}{*}{ Structure } & \multicolumn{4}{|c|}{ Relative abundance (peak height), $\%$} \\
\hline & & & & & $1-\mathrm{C}_{4} \mathrm{H}_{8}$ & $\mathrm{C}_{3} \mathrm{H}_{6}$ & $\mathrm{C}_{2} \mathrm{H}_{4}$ & $\begin{array}{c}\mathrm{CH}_{3} \mathrm{OH}(\mathrm{Rt}, \\
\min )^{\mathrm{f}}\end{array}$ \\
\hline 1 & 2.74 & 70 & $\mathrm{C}_{5} \mathrm{H}_{10}$ & & 0.31 & trace & trace & \\
\hline 2 & $\begin{array}{l}3.59 \\
3.65 \\
3.75 \\
\end{array}$ & $\begin{array}{l}78 \\
84 \\
84 \\
\end{array}$ & $\begin{array}{c}\mathrm{C}_{6} \mathrm{H}_{6} \\
\mathrm{C}_{6} \mathrm{H}_{12} \\
\mathrm{C}_{6} \mathrm{H}_{12} \\
\end{array}$ & Overlap with $\mathrm{CCl}_{4}$ & $\sqrt{ }$ & $\sqrt{ }$ & $\sqrt{ }$ & $\begin{array}{c}\sqrt{ }(3.92) \\
(3.96) \\
(4.04)\end{array}$ \\
\hline 3 & 4.89 & 98 & $\mathrm{C}_{7} \mathrm{H}_{14}$ & & 0.11 & trace & & $\operatorname{Trace}(4.73)$ \\
\hline 4 & 5.09 & 98 & $\mathrm{C}_{7} \mathrm{H}_{14}$ & & 0.07 & trace & & \\
\hline 5 & 5.75 & 92 & $\mathrm{C}_{7} \mathrm{H}_{8}$ & & 71.8 & 14.5 & 31.3 & $17.0(5.21)$ \\
\hline 6 & 5.93 & 112 & $\mathrm{C}_{8} \mathrm{H}_{16}$ & & 0.12 & trace & & \\
\hline 7 & 6.41 & & & & trace & trace & & \\
\hline 8 & 6.60 & 112 & $\mathrm{C}_{8} \mathrm{H}_{16}$ & & trace & trace & & \\
\hline 9 & 8.78 & 106 & $\mathrm{C}_{8} \mathrm{H}_{10}$ & & 1.75 & 0.65 & 14.6 & $1.56(7.04)$ \\
\hline 10 & 9.08 & 106 & $\mathrm{C}_{8} \mathrm{H}_{10}$ & & 16.49 & 5.24 & 11.0 & $11.9(7.22)$ \\
\hline 11 & 9.87 & 106 & $\mathrm{C}_{8} \mathrm{H}_{10}$ & & 17.56 & 5.26 & 9.50 & $10.3(7.76)$ \\
\hline 12 & 11.07 & 120 & $\mathrm{C}_{9} \mathrm{H}_{12}$ & & 2.46 & 2.17 & 0.59 & $0.24(8.58)$ \\
\hline 13 & 11.83 & 106 & $\mathrm{C}_{8} \mathrm{H}_{10}$ & & 0.06 & 0.02 & 0.60 & \\
\hline 14 & 12.11 & 120 & $\mathrm{C}_{9} \mathrm{H}_{12}$ & & 0.53 & 0.47 & 0.31 & $0.18(9.35)$ \\
\hline 15 & 12.38 & & & & 0.61 & 0.20 & 4.03 & $0.71(9.6)$ \\
\hline 16 & 13.98 & 120 & $\mathrm{C}_{9} \mathrm{H}_{12}$ & & 0.64 & 0.26 & 4.95 & $0.79(10.0)$ \\
\hline 17 & 12.66 & & & & 0.81 & 0.23 & 0.76 & $1.68(9.8)$ \\
\hline 18 & 13.50 & 120 & $\mathrm{C}_{9} \mathrm{H}_{12}$ & & 6.20 & 1.85 & 3.90 & $7.79(10.5)$ \\
\hline 19 & 14.44 & & & & 3.71 & 0.92 & 1.61 & $2.58(11.25)$ \\
\hline 20 & 14.06 & 134 & $\mathrm{C}_{10} \mathrm{H}_{14}$ & & 0.06 & & & \\
\hline 21 & 14.14 & 134 & $\mathrm{C}_{10} \mathrm{H}_{14}$ & & 1.52 & 0.30 & 0.25 & $0.08(10.95)$ \\
\hline 22 & 14.55 & 134 & $\mathrm{C}_{10} \mathrm{H}_{14}$ & & 1.54 & 0.73 & 0.85 & \\
\hline 23 & 14.63 & 134 & $\mathrm{C}_{10} \mathrm{H}_{14}$ & & 0.18 & 0.21 & & \\
\hline 24 & 15.07 & 134 & $\mathrm{C}_{10} \mathrm{H}_{14}$ & & 1.96 & 1.42 & 1.31 & \\
\hline
\end{tabular}




\begin{tabular}{|c|c|c|c|c|c|c|c|c|}
\hline 25 & 14.81 & 118 & $\mathrm{C}_{9} \mathrm{H}_{10}$ & & 1.25 & 0.27 & 1.83 & $0.31(11.57)$ \\
\hline 26 & 15.48 & \multirow{3}{*}{134} & \multirow{3}{*}{$\mathrm{C}_{10} \mathrm{H}_{14}$} & \multirow{3}{*}{$\left(\mathrm{C}_{2} \mathrm{H}_{5}\right)_{2}$} & 0.17 & 0.06 & 0.4 & $0.1(12.12)$ \\
\hline 27 & 15.67 & & & & 0.11 & & 0.2 & \\
\hline 28 & 15.80 & & & & & & 0.5 & \\
\hline 29 & 16.00 & 134 & $\mathrm{C}_{10} \mathrm{H}_{14}$ & & 0.15 & 0.05 & & \\
\hline 30 & & 134 & $\mathrm{C}_{10} \mathrm{H}_{14}$ & & & & & $0.2(12.84)$ \\
\hline $31^{d}$ & 16.25 & & $\mathrm{C}_{2} \mathrm{Cl}_{6}$ & Impurity from $\mathrm{CCl}_{4}$ & $\sqrt{ }$ & $\sqrt{ }$ & $\sqrt{ }$ & \\
\hline 32 & 16.39 & 132 & $\mathrm{C}_{10} \mathrm{H}_{12}$ & & 0.58 & 0.16 & 1.05 & $0.25(12.91)$ \\
\hline 33 & 16.55 & 132 & $\mathrm{C}_{10} \mathrm{H}_{12}$ & & 2.36 & 0.72 & 2.11 & $0.43(13.09)$ \\
\hline 34 & 16.77 & 134 & $\mathrm{C}_{10} \mathrm{H}_{14}$ & & 0.13 & 0.03 & 0.4 & $0.15(13.3)$ \\
\hline 35 & 16.99 & 146 & $\mathrm{C}_{11} \mathrm{H}_{14}$ & & 0.14 & 0.04 & 0.15 & $\operatorname{trace}(13.49)$ \\
\hline 36 & 17.22 & 146 & $\mathrm{C}_{11} \mathrm{H}_{14}$ & & 0.89 & 0.12 & 0.55 & $0.15(13.68)$ \\
\hline 37 & 17.28 & 148 & $\mathrm{C}_{11} \mathrm{H}_{16}$ & & $\sqrt{ }$ & $\sqrt{ }$ & $\sqrt{ }$ & \\
\hline 38 & 17.36 & 150 & $\mathrm{C}_{11} \mathrm{H}_{18}$ & & 0.26 & Trace & & \\
\hline 39 & 17.46 & 148 & $\mathrm{C}_{11} \mathrm{H}_{16}$ & & 0.41 & & & \\
\hline 40 & 17.61 & \multirow{2}{*}{134} & \multirow{2}{*}{$\mathrm{C}_{10} \mathrm{H}_{14}$} & & 1.24 & 0.35 & 0.77 & $2.66(14.0)$ \\
\hline 41 & 17.72 & & & & 1.98 & 0.13 & 0.23 & $0.90(14.1)$ \\
\hline 42 & 17.65 & 148 & $\mathrm{C}_{11} \mathrm{H}_{16}$ & & $\sqrt{ }$ & $\sqrt{ }$ & $\sqrt{ }$ & \\
\hline 43 & 17.88 & 164 & $\mathrm{C}_{12} \mathrm{H}_{20}$ & isomers & 0.11 & & & \\
\hline 44 & 18.10 & 148 & $\mathrm{C}_{11} \mathrm{H}_{16}$ & & 0.23 & 0.14 & & \\
\hline 45 & 18.24 & 148 & $\mathrm{C}_{11} \mathrm{H}_{16}$ & $\begin{array}{l}\left.-1 \mathrm{CH}_{3}\right)_{2} \\
\text { p with } 45\end{array}$ & 0.68 & & & \\
\hline 46 & 18.27 & 146 & $\mathrm{C}_{11} \mathrm{H}_{14}$ & & $\sqrt{ }$ & & & \\
\hline 47 & 18.25 & 148 & $\mathrm{C}_{11} \mathrm{H}_{16}$ & & & 0.51 & & \\
\hline
\end{tabular}




\begin{tabular}{|c|c|c|c|c|c|c|c|c|}
\hline 48 & 18.25 & 148 & $\mathrm{C}_{11} \mathrm{H}_{16}$ & $\overbrace{\frac{1}{d}\left(\mathrm{C}_{2} \mathrm{H}_{3}\right)_{2}}^{\mathrm{C}^{2}}$ & & & 0.72 & \\
\hline 49 & 18.25 & 146 & $\mathrm{C}_{11} \mathrm{H}_{14}$ & & & & & $0.19(14.62)$ \\
\hline 50 & 18.48 & 148 & $\mathrm{C}_{11} \mathrm{H}_{16}$ & & 0.18 & 0.24 & & \\
\hline 51 & 18.53 & 132 & $\mathrm{C}_{10} \mathrm{H}_{12}$ & & 0.76 & 0.2 & 1.42 & $0.48(14.90)$ \\
\hline 52 & 18.69 & 148 & $\mathrm{C}_{11} \mathrm{H}_{16}$ & & 0.09 & 0.03 & & $0.19(15)$ \\
\hline 53 & 18.85 & 132 & $\mathrm{C}_{10} \mathrm{H}_{12}$ & & 1.46 & 0.54 & 1.47 & $0.17(15.19)$ \\
\hline 54 & 19.19 & 148 & $\mathrm{C}_{11} \mathrm{H}_{16}$ & & 0.40 & 0.35 & 0.39 & \\
\hline 55 & 19.51 & 128 & $\mathrm{C}_{10} \mathrm{H}_{8}$ & & 100 & 100 & 100 & $100(15.74)$ \\
\hline 56 & 19.79 & \multirow{3}{*}{146} & \multirow{3}{*}{$\mathrm{C}_{11} \mathrm{H}_{14}$} & & 0.32 & 0.10 & 0.50 & \\
\hline 57 & 19.92 & & & & 0.38 & 0.14 & 0.30 & $0.33(16.09)$ \\
\hline 58 & 20.04 & & & & 0.43 & 0.13 & & $0.16(16.19)$ \\
\hline 59 & 20.15 & 148 & $\mathrm{C}_{11} \mathrm{H}_{16}$ & & 0.13 & Trace & 0.5 & \\
\hline 60 & & 134 & $\mathrm{C}_{9} \mathrm{H}_{10} \mathrm{O}$ & & & & & $27.8(16.35)$ \\
\hline 61 & 20.54 & 146 & $\mathrm{C}_{11} \mathrm{H}_{14}$ & Overlap with 62 & $\sqrt{ }$ & $\sqrt{ }$ & & \\
\hline 62 & 20.59 & 170 & $\mathrm{C}_{12} \mathrm{H}_{26}$ & $\begin{array}{c}\mathrm{C}_{\mathrm{n}} \mathrm{H}_{2 \mathrm{n}+2} \\
\text { Overlap with } 61,63\end{array}$ & 2.05 & 1.45 & 5.54 & $0.42(16.76)$ \\
\hline 63 & 20.59 & 162 & $\mathrm{C}_{12} \mathrm{H}_{18}$ & Overlap with 62 & $\sqrt{ }$ & & & \\
\hline 64 & 20.80 & 146 & $\mathrm{C}_{11} \mathrm{H}_{14}$ & & 0.25 & 0.06 & & Trace(17.01) \\
\hline 65 & 20.96 & \multirow{2}{*}{162} & \multirow{2}{*}{$\mathrm{C}_{12} \mathrm{H}_{18}$} & & 0.12 & & & \\
\hline 66 & 21.06 & & & & 0.10 & & & \\
\hline 67 & 21.38 & 160 & $\mathrm{C}_{12} \mathrm{H}_{16}$ & isomers & 0.15 & 0.05 & & \\
\hline 68 & 21.50 & 162 & $\mathrm{C}_{12} \mathrm{H}_{18}$ & & 0.22 & & & \\
\hline 68 & 21.65 & 162 & $\mathrm{C}_{12} \mathrm{H}_{18}$ & & 0.13 & 0.15 & & \\
\hline 70 & 21.83 & 162 & $\mathrm{C}_{12} \mathrm{H}_{18}$ & & 0.17 & 0.06 & & \\
\hline 71 & 22.54 & 198 & $\mathrm{C}_{14} \mathrm{H}_{30}$ & $\mathrm{C}_{\mathrm{n}} \mathrm{H}_{2 \mathrm{n}+2}$ & 0.07 & 0.17 & 0.3 & \\
\hline 72 & & 148 & $\mathrm{C}_{11} \mathrm{H}_{16}$ & & & & & $0.27(18.65)$ \\
\hline 73 & 22.68 & 141 & $\mathrm{C}_{11} \mathrm{H}_{10}$ & & 15.0 & 14.7 & 16.4 & $53.5(18.9)$ \\
\hline
\end{tabular}




\begin{tabular}{|c|c|c|c|c|c|c|c|c|}
\hline 74 & 23.15 & & & & 36.2 & 30.2 & 43.9 & $66.8(19.3)$ \\
\hline 75 & 23.43 & 184 & $\mathrm{C}_{13} \mathrm{H}_{28}$ & $\mathrm{C}_{\mathrm{n}} \mathrm{H}_{2 \mathrm{n}+2}$ & 0.21 & 0.27 & 0.73 & Trace(19.53) \\
\hline 76 & 24.25 & & $\mathrm{C}_{\mathrm{n}} \mathrm{H}_{2 \mathrm{n}+2}$ & $\mathrm{C}_{\mathrm{n}} \mathrm{H}_{2 \mathrm{n}+2}$ & 0.11 & 0.15 & 0.46 & $0.14(20.35)$ \\
\hline 77 & 24.43 & 174 & $\mathrm{C}_{13} \mathrm{H}_{18}$ & & 0.29 & & & \\
\hline 78 & 24.89 & 154 & $\mathrm{C}_{12} \mathrm{H}_{10}$ & & 0.07 & 0.05 & 0.10 & $0.26(21.05)$ \\
\hline 79 & 25.15 & 158 & $\mathrm{C}_{12} \mathrm{H}_{14}$ & & 0.22 & 0.07 & & \\
\hline 80 & 25.45 & 156 & $\mathrm{C}_{12} \mathrm{H}_{12}$ & & 0.84 & 0.50 & 22.5 & $0.63(21.57)$ \\
\hline 81 & 26.05 & & & & 1.63 & 1.09 & 7.18 & $10.8(22.19)$ \\
\hline 82 & 26.56 & 156 & $\mathrm{C}_{12} \mathrm{H}_{12}$ & & 1.23 & 0.91 & 5.5 & $12.2(22.70)$ \\
\hline 83 & 26.90 & & & & 0.21 & 0.19 & 0.96 & $2.47(23.05)$ \\
\hline 84 & 26.78 & 160 & $\mathrm{C}_{12} \mathrm{H}_{16}$ & & 0.10 & & & \\
\hline 85 & 27.11 & 170 & $\mathrm{C}_{13} \mathrm{H}_{14}$ & & 4.62 & 14.4 & 3.05 & \\
\hline 86 & 27.55 & 154 & $\mathrm{C}_{12} \mathrm{H}_{10}$ & & 0.15 & 0.10 & 0.80 & $3.21(23.72)$ \\
\hline 87 & 27.70 & 156 & $\mathrm{C}_{12} \mathrm{H}_{12}$ & & 1.37 & 0.70 & 1.34 & \\
\hline 88 & 27.94 & 172 & $\mathrm{C}_{13} \mathrm{H}_{16}$ & & 1.21 & 0.23 & & \\
\hline 89 & 28.00 & 170 & $\mathrm{C}_{13} \mathrm{H}_{14}$ & & & & 1.35 & \\
\hline $90^{e}$ & 28.39 & 206 & $\mathrm{C}_{14} \mathrm{H}_{22} \mathrm{O}$ & & 2.56 & 4.31 & 13.2 & $9.61(24.49)$ \\
\hline 91 & 28.61 & 212 & $\mathrm{C}_{15} \mathrm{H}_{32}$ & $\mathrm{C}_{\mathrm{n}} \mathrm{H}_{2 \mathrm{n}+2}$ & 0.14 & 0.14 & 0.42 & $1.17(24.71)$ \\
\hline 92 & 28.69 & $\begin{array}{l}212 \\
170\end{array}$ & $\begin{array}{l}\mathrm{C}_{15} \mathrm{H}_{32} \\
\mathrm{C}_{13} \mathrm{H}_{14}\end{array}$ & & 0.34 & 0.56 & 3.27 & $1.12(24.79)$ \\
\hline 93 & 28.84 & 170 & $\mathrm{C}_{13} \mathrm{H}_{14}$ & & trace & trace & 0.57 & \\
\hline 94 & 28.89 & & $\mathrm{C}_{\mathrm{n}} \mathrm{H}_{2 \mathrm{n}+2}$ & $\mathrm{C}_{\mathrm{n}} \mathrm{H}$ & & 0.09 & trace & $0.76(24.98)$ \\
\hline 95 & 29.05 & & $\mathrm{C}_{\mathrm{n}} \mathrm{H}_{2 \mathrm{n}+2}$ & $\mathrm{C}_{\mathrm{n}} \mathrm{H}_{2 \mathrm{n}+2}$ & & 0.07 & & $0.42(25.1)$ \\
\hline 96 & 29.14 & 184 & $\mathrm{C}_{14} \mathrm{H}_{16}$ & & 1.11 & & & \\
\hline 97 & 29.25 & 170 & $\mathrm{C}_{13} \mathrm{H}_{14}$ & & & & 0.60 & $0.87(25.35)$ \\
\hline
\end{tabular}




\begin{tabular}{|c|c|c|c|c|c|c|c|c|}
\hline 98 & 29.48 & 184 & $\mathrm{C}_{14} \mathrm{H}_{16}$ & 11 & 0.25 & 0.19 & & \\
\hline 99 & 29.65 & 172 & $\mathrm{C}_{13} \mathrm{H}_{16}$ & & 0.21 & 0.16 & & \\
\hline 100 & 29.65 & 184 & $\mathrm{C}_{14} \mathrm{H}_{16}$ & & & $\sqrt{ }$ & & \\
\hline 101 & 29.65 & 170 & $\mathrm{C}_{13} \mathrm{H}_{14}$ & & & $\sqrt{ }$ & 0.51 & $2.3(25.80)$ \\
\hline 102 & 29.75 & & $\mathrm{C}_{\mathrm{n}} \mathrm{H}_{2 \mathrm{n}+2}$ & $\mathrm{C}_{\mathrm{n}} \mathrm{H}_{2 \mathrm{n}+2}$ & 0.19 & 0.27 & 0.81 & $1.0(25.85)$ \\
\hline 103 & 29.95 & 166 & $\mathrm{C}_{13} \mathrm{H}_{10}$ & & 0.82 & 1.32 & 3.72 & $6.49(26.1)$ \\
\hline 104 & 30.07 & 184 & $\mathrm{C}_{14} \mathrm{H}_{16}$ & & & & 0.63 & \\
\hline 105 & 30.12 & 184 & $\mathrm{C}_{14} \mathrm{H}_{16}$ & & 0.04 & 0.05 & & \\
\hline 106 & 30.22 & 168 & $\mathrm{C}_{13} \mathrm{H}_{12}$ & & 0.23 & 0.11 & 1.47 & $2.81(26.39)$ \\
\hline 107 & 30.42 & 172 & $\mathrm{C}_{13} \mathrm{H}_{16}$ & & 0.31 & 0.14 & & \\
\hline 108 & 30.42 & 168 & $\mathrm{C}_{13} \mathrm{H}_{12}$ & & & & 0.31 & $0.91(26.59)$ \\
\hline 109 & 30.64 & 184 & $\mathrm{C}_{14} \mathrm{H}_{16}$ & & & & 0.8 & \\
\hline 110 & 30.97 & 226 & $\mathrm{C}_{16} \mathrm{H}_{34}$ & $\mathrm{C}_{\mathrm{n}} \mathrm{H}_{2 \mathrm{n}+2}$ & 0.21 & trace & trace & $1.06(27.09)$ \\
\hline 111 & 31.07 & 184 & $\mathrm{C}_{14} \mathrm{H}_{16}$ & & & & 0.70 & \\
\hline 112 & 31.41 & 166 & $\mathrm{C}_{13} \mathrm{H}_{10}$ & & 8.94 & 3.1 & 8.10 & $5.73(27.55)$ \\
\hline 113 & 31.49 & 184 & $\mathrm{C}_{14} \mathrm{H}_{16}$ & & & & 0.82 & \\
\hline 114 & 31.61 & 182 & $\mathrm{C}_{14} \mathrm{H}_{14}$ & & 0.15 & Trace & 0.7 & \\
\hline 115 & 32.75 & 180 & $\mathrm{C}_{14} \mathrm{H}_{12}$ & & 0.08 & 0.1 & 0.4 & $1.1(28.93)$ \\
\hline 116 & 33.00 & 180 & $\mathrm{C}_{14} \mathrm{H}_{12}$ & & 0.09 & 0.1 & 0.53 & $0.80(29.18)$ \\
\hline
\end{tabular}




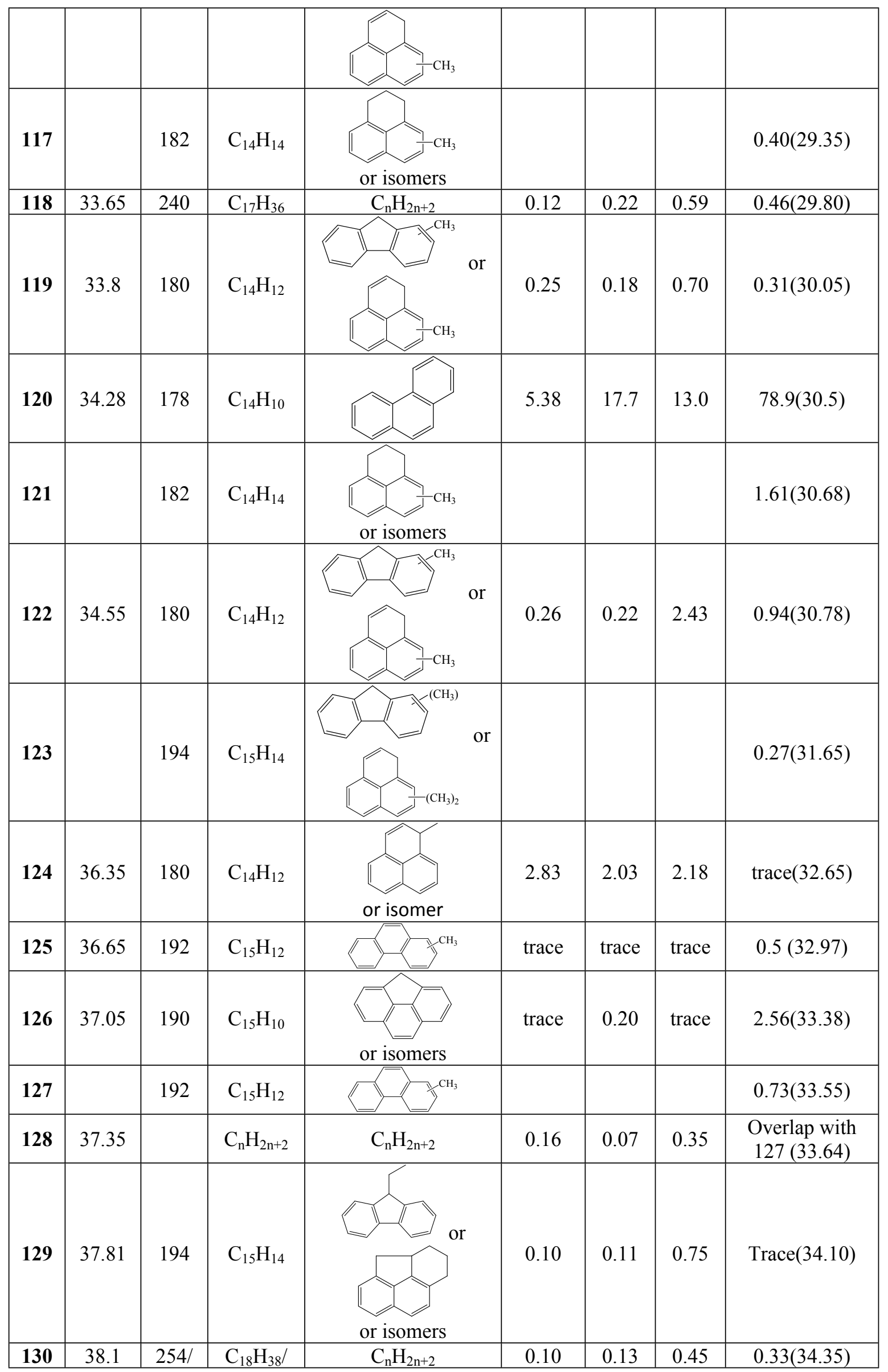




\begin{tabular}{|c|c|c|c|c|c|c|c|c|}
\hline & & 268 & $\mathrm{C}_{19} \mathrm{H}_{40}$ & & & & & \\
\hline 131 & 38.9 & & $\mathrm{C}_{\mathrm{n}} \mathrm{H}_{2 \mathrm{n}+2}$ & $\mathrm{C}_{\mathrm{n}} \mathrm{H}_{2 \mathrm{n}+2}$ & 0.10 & 0.12 & 0.35 & $0.13(35.2)$ \\
\hline 132 & 39.57 & 194 & $\mathrm{C}_{15} \mathrm{H}_{14}$ & & 0.42 & 0.23 & 0.91 & $0.35(35.94)$ \\
\hline 133 & 40.75 & 202 & $\mathrm{C}_{16} \mathrm{H}_{10}$ & & 0.53 & 6.81 & 1.67 & $72.4(37.2)$ \\
\hline 134 & 41.03 & $\begin{array}{l}212 \\
248\end{array}$ & $\begin{array}{c}\mathrm{C}_{16} \mathrm{H}_{20} \\
\mathrm{C}_{14} \mathrm{H}_{8} \mathrm{Cl}_{2}\end{array}$ & & 1.41 & 1.04 & 1.3 & $3.1(37.43)$ \\
\hline 135 & 42.97 & & & & trace & 0.06 & trace & $0.41(39.39)$ \\
\hline 136 & 43.21 & 216 & $C$ & & trace & 0.05 & trace & $0.20(39.65)$ \\
\hline 137 & 43.36 & & & & trace & 0.06 & trace & $0.72(39.80)$ \\
\hline 138 & 43.49 & 220 & $\mathrm{C}_{17} \mathrm{H}_{16}$ & & trace & 0.1 & trace & $0.18(39.90)$ \\
\hline 139 & 31.24 & 224 & $\mathrm{C}_{17} \mathrm{H}_{20}$ & & Trace & Trace & Trace & Trace(27.40) \\
\hline 140 & 44.40 & 366 & $\mathrm{C}_{29} \mathrm{H}_{18}$ & & trace & 0.08 & 0.37 & $\operatorname{trace}(40.77)$ \\
\hline 141 & 45.40 & 344 & $\mathrm{C}_{27} \mathrm{H}_{20}$ & & & trace & trace & \\
\hline
\end{tabular}

a. Rt, retention time for soluble coke of 1-butene, propene and ethene. The GC condition was 50 ${ }^{\circ} \mathrm{C}$ for $5 \mathrm{~min}$, ramped to $250^{\circ} \mathrm{C}$ with a rate $5^{\circ} \mathrm{C} / \mathrm{min}$, and kept for $5 \mathrm{~min}$.

b. MW, molecular weight;

c. MF, molecular formula;

d. Rt, retention time for soluble coke of methanol. The $\mathrm{GC}$ condition was $60{ }^{\circ} \mathrm{C}$ for $2 \mathrm{~min}$, ramped to $250^{\circ} \mathrm{C}$ with a rate $5^{\circ} \mathrm{C} / \mathrm{min}$, and kept for $5 \mathrm{~min}$.

e. Impurity from $\mathrm{CCl}_{4}$;

f. Antioxidant in plastic (centrifuge tube). 

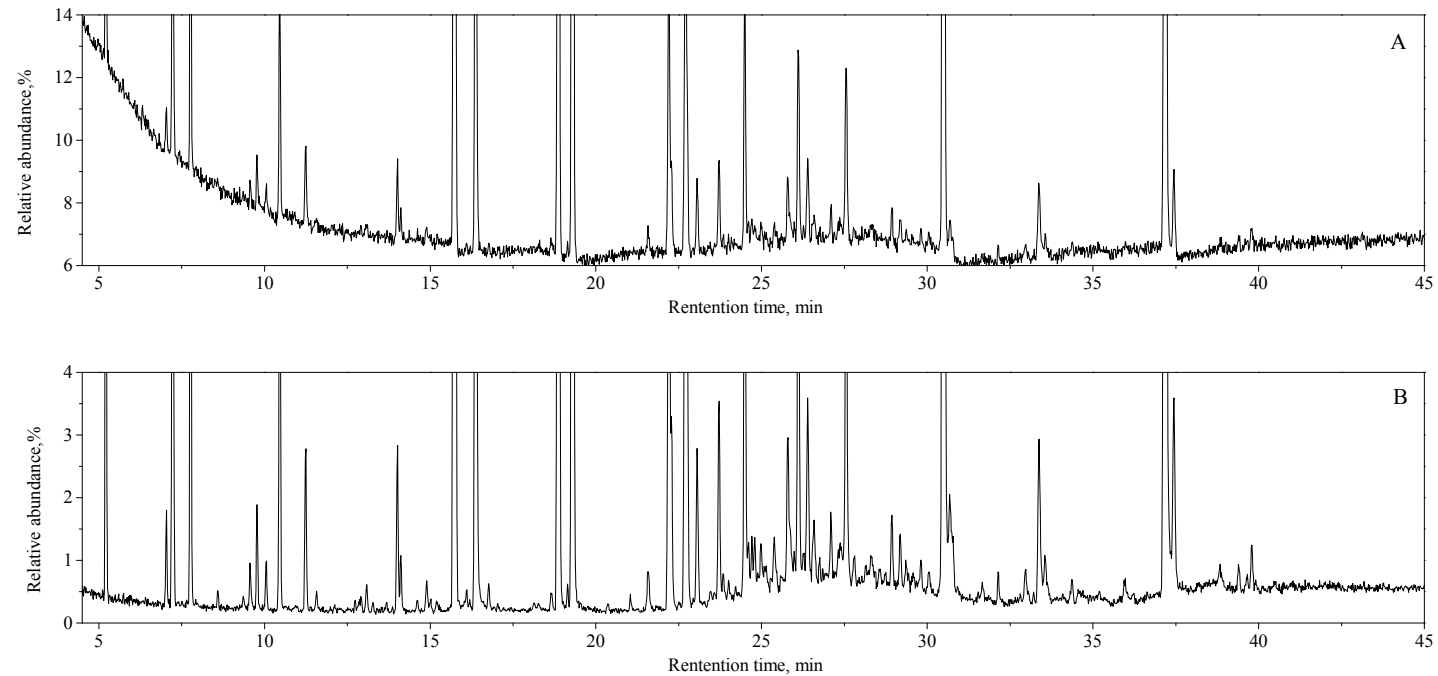

Figure S1. Original (A) and denoised (B) GC-MS total ion chromatogram of methanol feed. 

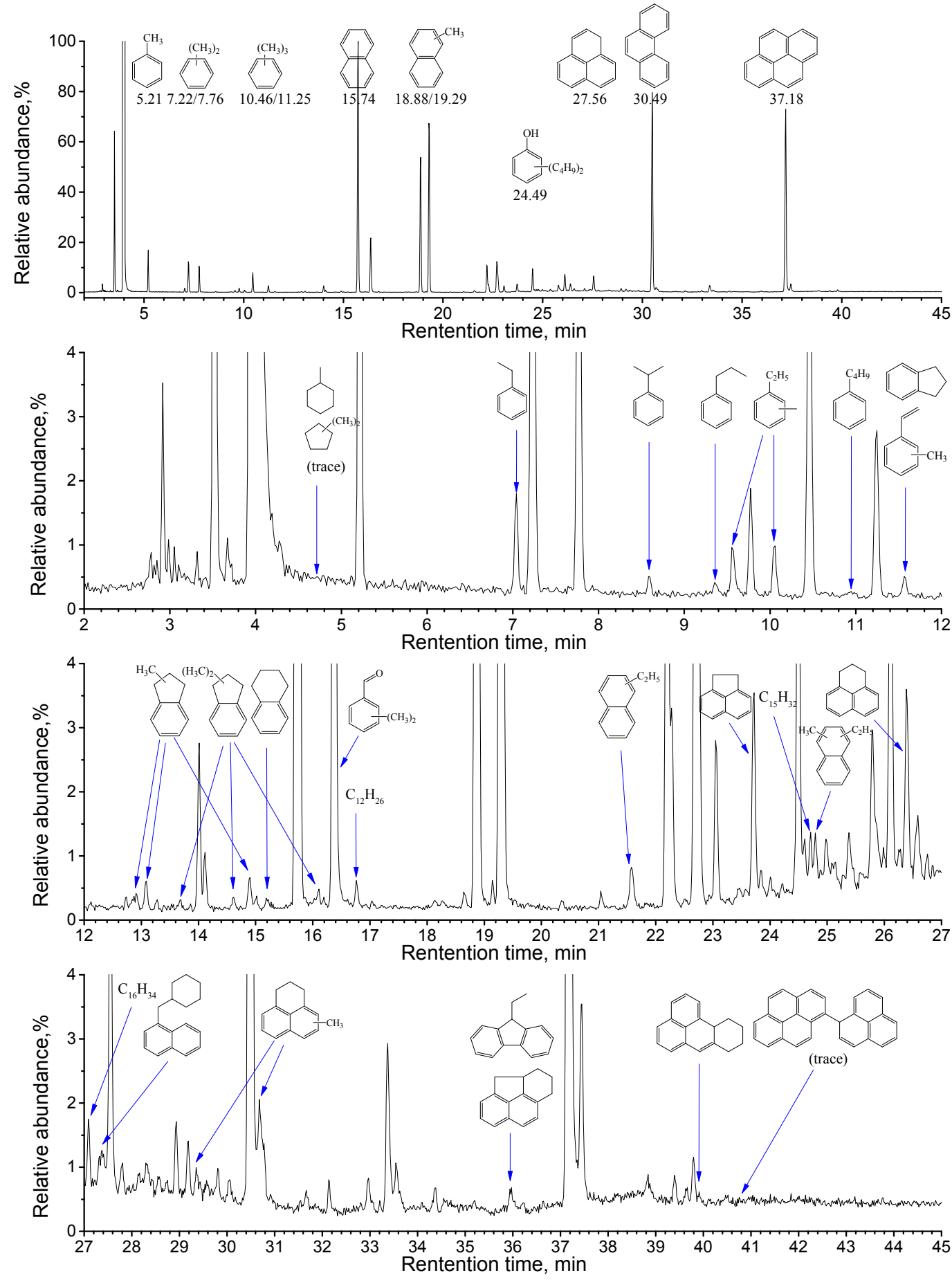

Figure S2. GC-MS total ion chromatogram and identified confined compounds from methanol feed. 

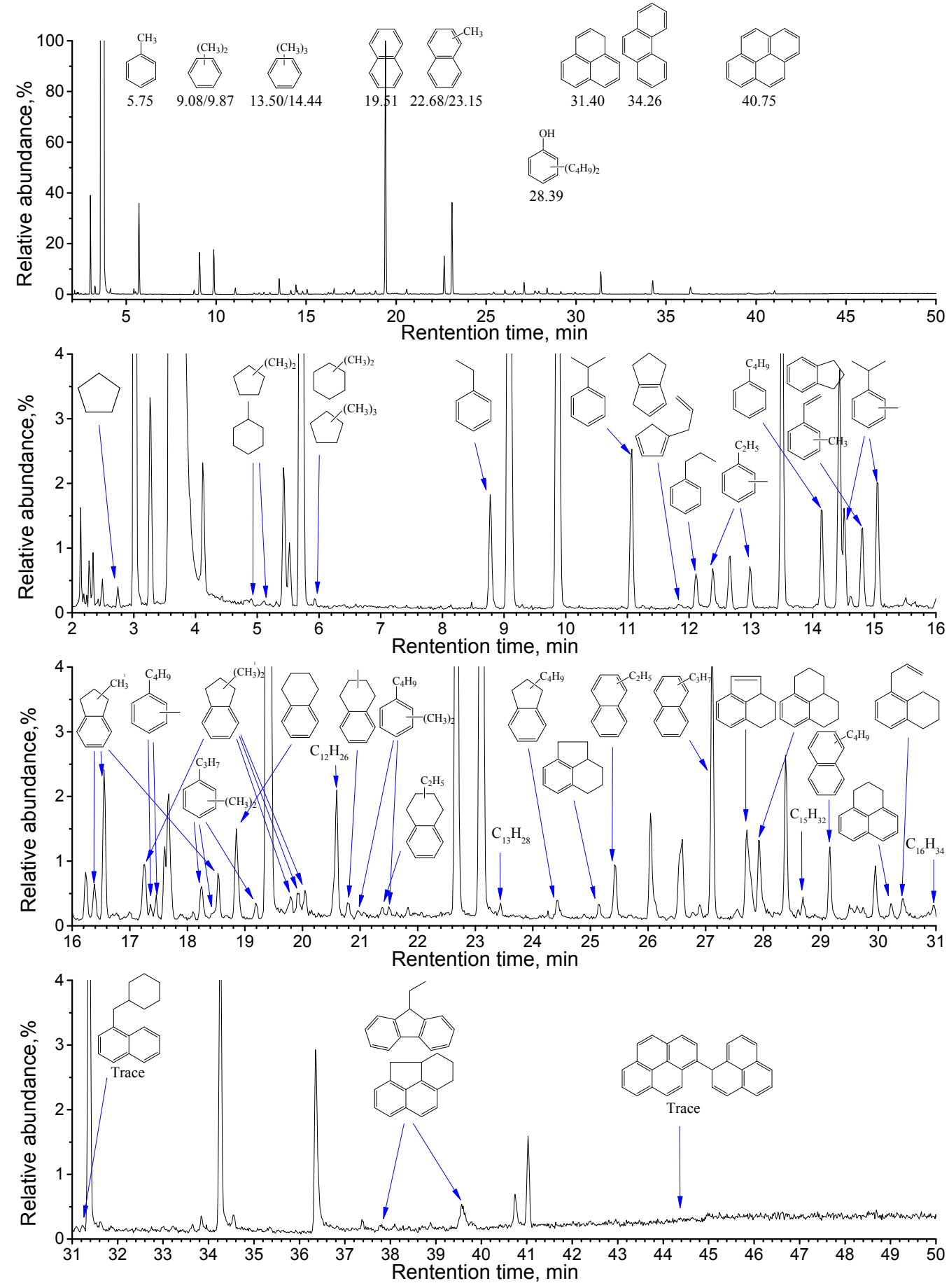

Figure S3. GC-MS total ion chromatogram and identified confined compounds from 1-butene feed. 

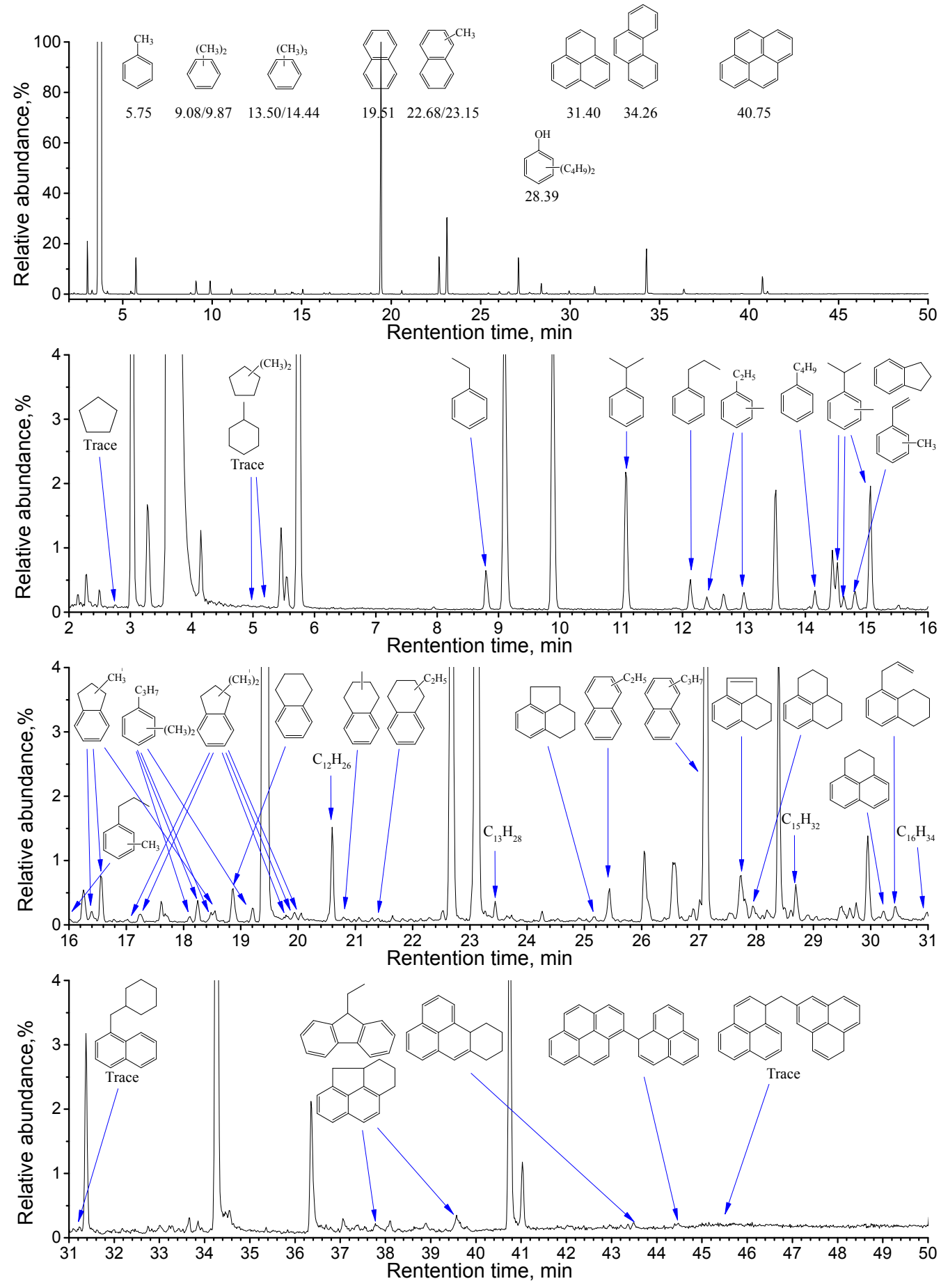

Figure S4. GC-MS total ion chromatogram and identified confined compounds from propene feed. 

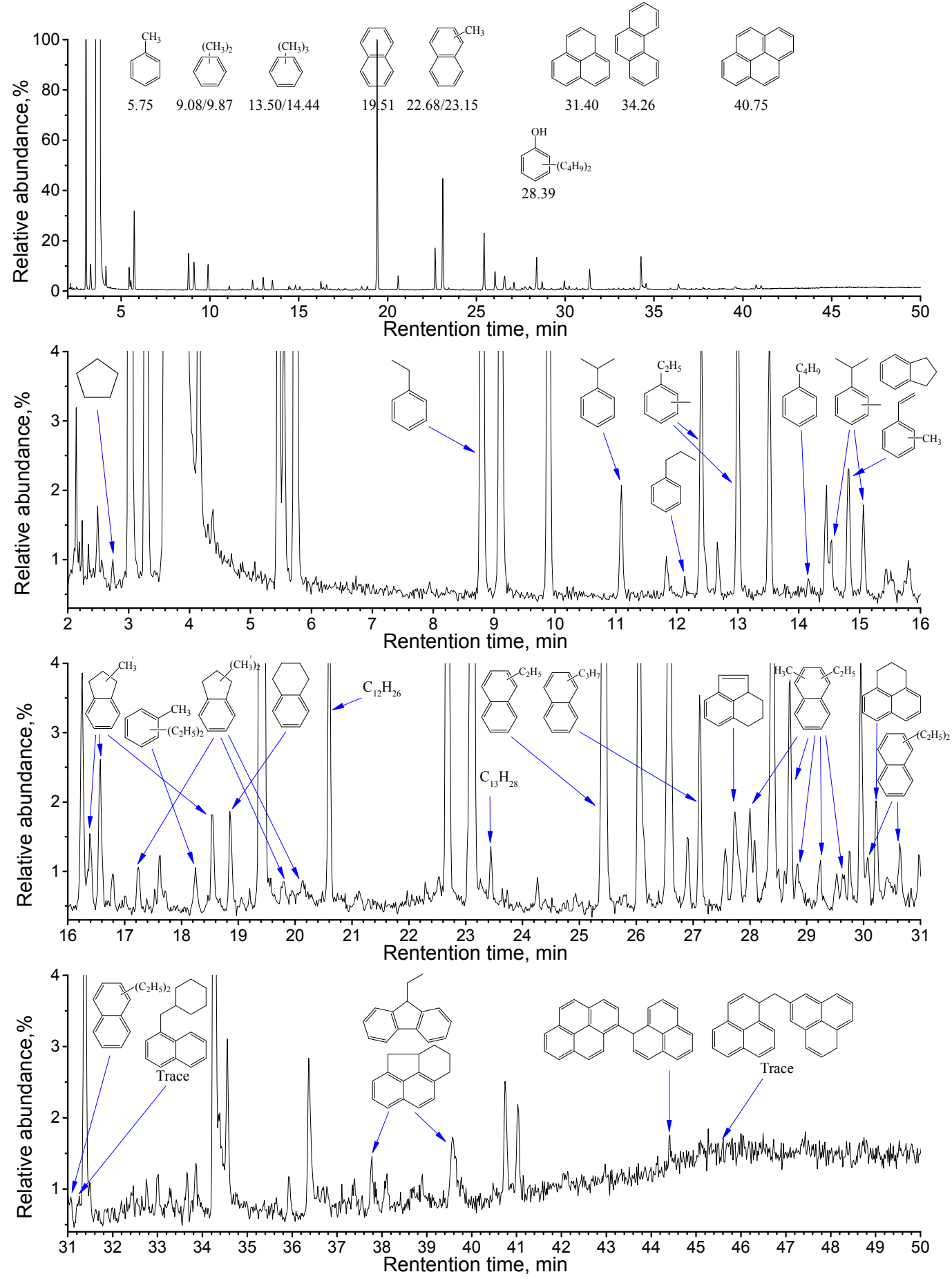

Figure S5. GC-MS total ion chromatogram and identified confined compounds from ethene feed. 

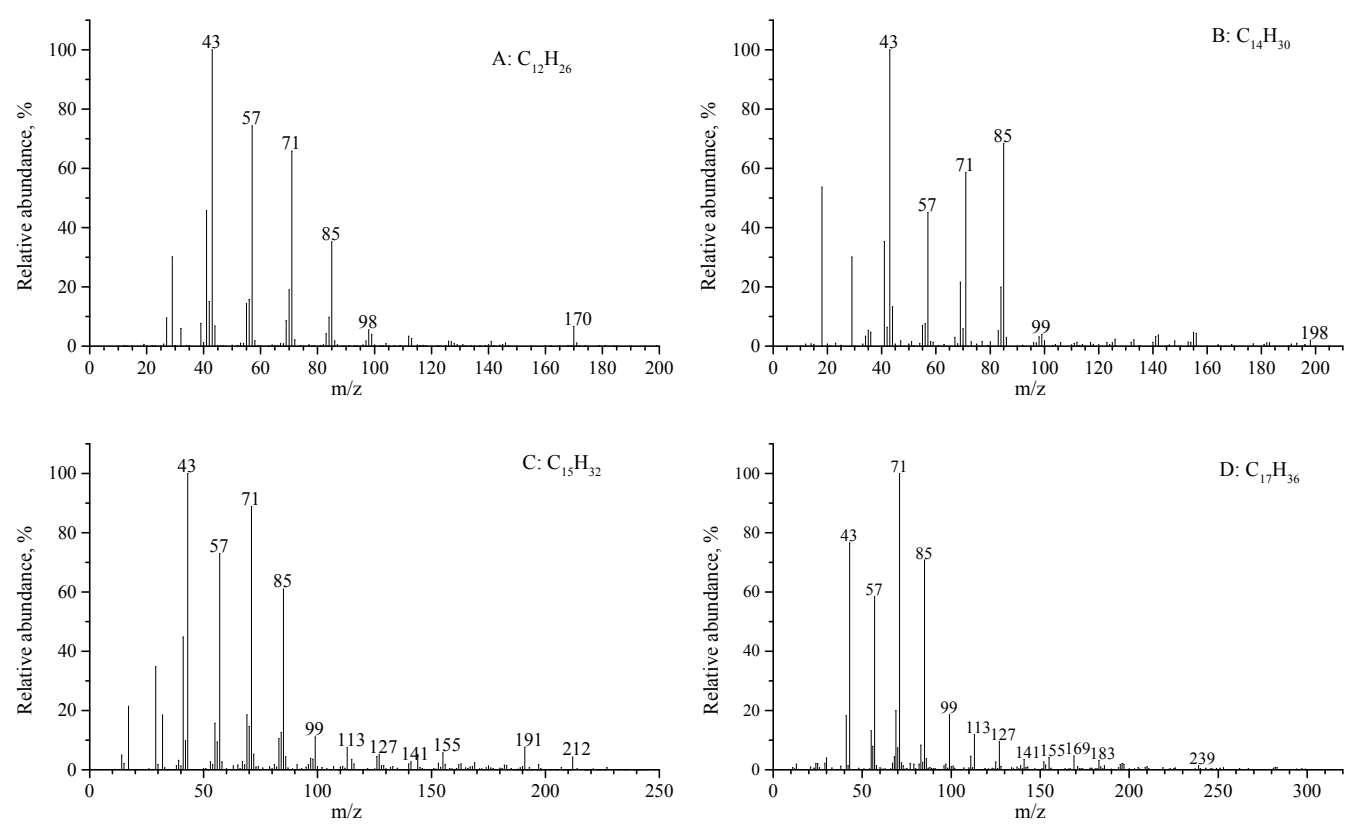

Figure S6. Typical fragment ion spectra of detected alkanes. A: $\mathrm{C}_{12} \mathrm{H}_{26}$ (No. 62); B: $\mathrm{C}_{14} \mathrm{H}_{30}$ (No.

$$
\text { 71); } \mathrm{C}: \mathrm{C}_{15} \mathrm{H}_{32} \text { (No. 91); and D: } \mathrm{C}_{17} \mathrm{H}_{36} \text { (No. 188). }
$$

As showed in Figure S6, fragment ions at $\mathrm{m} / \mathrm{z}$ of $43,57,71,85$, etc., are observed on compounds No. 62, 71, 75, 91, 110, 118, 130, etc. Molecular ions are observed at 170,198, 212, etc. These characteristics indicated the compounds are $\mathrm{C}_{12-19}$ alkanes. 

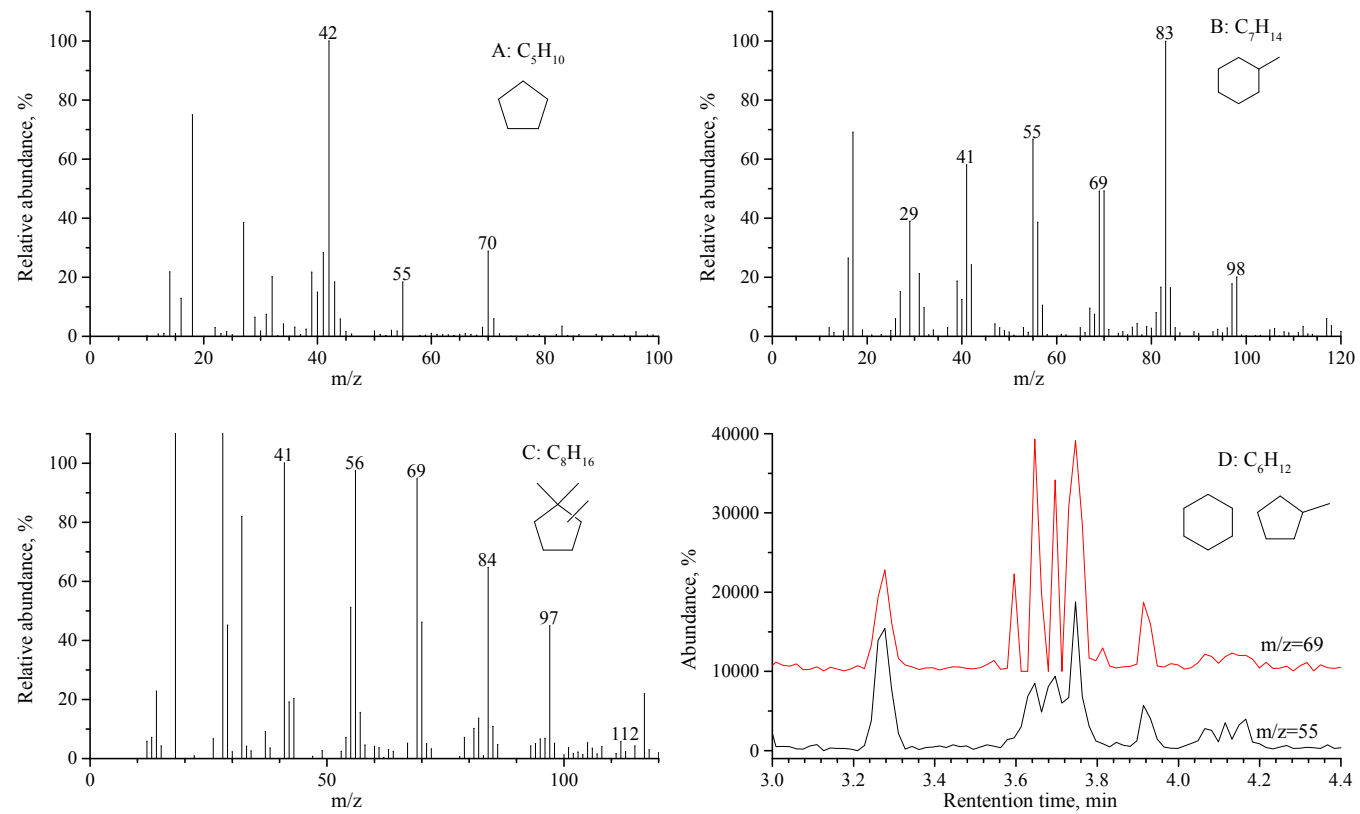

Figure S7. Typical fragment ion spectra of detected cycloalkanes. A: $\mathrm{C}_{5} \mathrm{H}_{10}$ (No. 1); B: $\mathrm{C}_{7} \mathrm{H}_{14}$ (No. 3); $\mathrm{C}: \mathrm{C}_{8} \mathrm{H}_{16}$ (No. 6); and D: Ion chromatogram of $\mathrm{C}_{6} \mathrm{H}_{12}$ (No. 2).

Typical fragment ion spectra of detected cycloalkanes (No. 1 4, 5-8) were showed in Figure S7. Typical fragment ions are observed at $\mathrm{m} / \mathrm{z}$ of 41(42), 55(56), $69(70), 83(84)$, etc. Signal of $\mathrm{C}_{6} \mathrm{H}_{12}$ overlaps with that of $\mathrm{CCl}_{4}$. However, feature ion fragments at $\mathrm{m} / \mathrm{z} 69$ and 55 between 3.5-4 min indicate the presence of cyclohexane and methylcyclopentane (Figure S7 D). Cycloalkanes are very low relative abundance. 

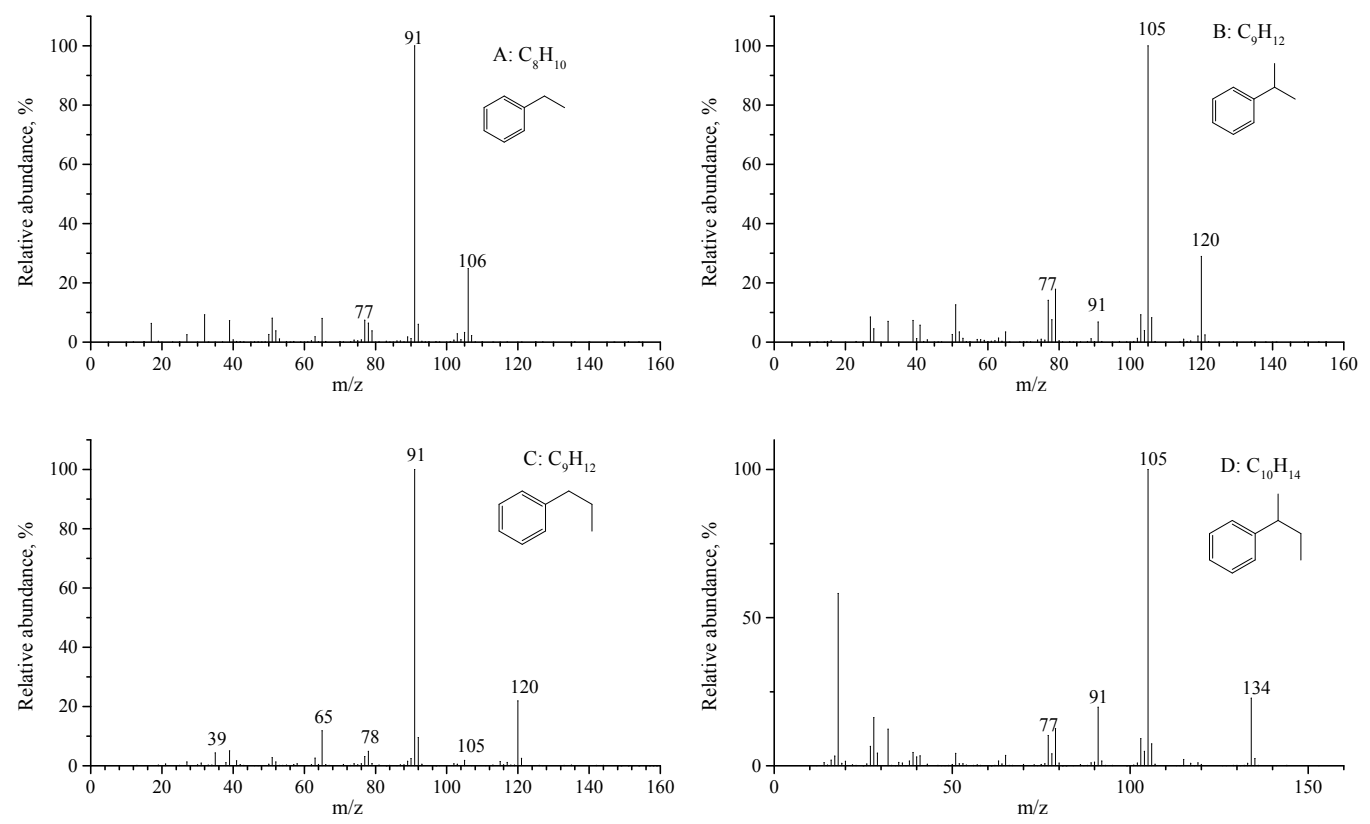

Figure S8. Typical fragment ion spectra of detected alkylbenzenes. A: $\mathrm{C}_{8} \mathrm{H}_{10}$ (No. 9); B: $\mathrm{C}_{9} \mathrm{H}_{12}$ (No. 12); $\mathrm{C}: \mathrm{C}_{9} \mathrm{H}_{12}$ (No. 14); and D: $\mathrm{C}_{10} \mathrm{H}_{14}$ (No. 21).

Typical fragment ion spectra of detected alkylbenzenes were showed in Figure S8. The abundance at $\mathrm{m} / \mathrm{z}=106$ of ethylbenzene is about $25 \%$, while that of dimethylbenzene is about $65 \%$. Moreover, the relative abundance of ethylbenzene from ethene feed comes to $14.6 \%$ while the values are much lower for other feeds. These differences favor the identification of ethylbenzene from $\mathrm{C}_{8} \mathrm{H}_{10}$ isomers. The relative low abundance of molecular ion is also one of the features to distinguish other $\mathrm{C}_{2+}$ side chain compounds, such as methylethylbenzene, isopropylbenzene, propylbenzene, isobutylbenzene, etc., from polymethylbenzene isomers. The cracking at $\beta \mathrm{C}-\mathrm{C}$ of side chain is a feature of $\mathrm{C}_{3+}$ side chain compounds. Feed also favors the identification of isomers. 

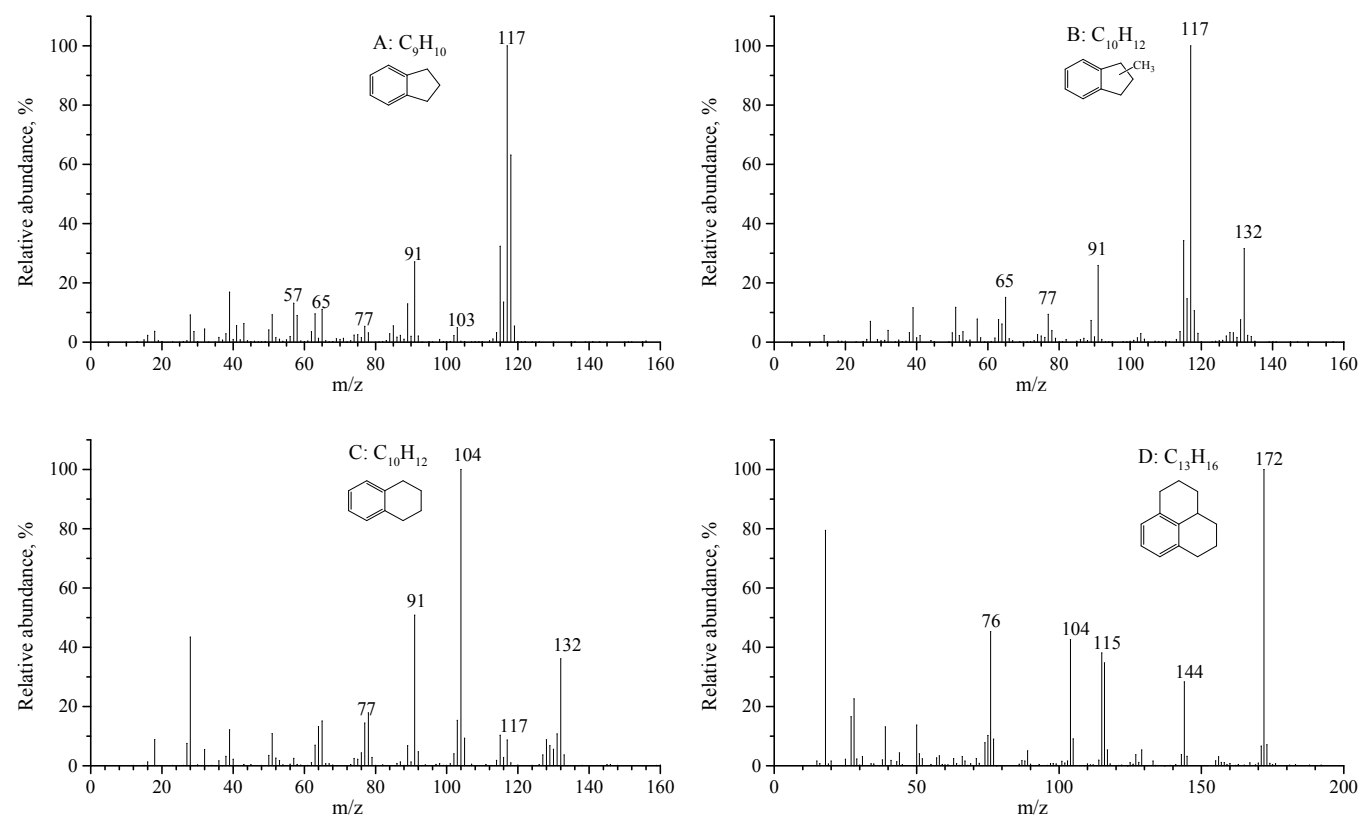

Figure S9. Typical fragment ion spectra of detected partially saturated bicyclic and tricyclic compounds. A: $\mathrm{C}_{9} \mathrm{H}_{10}$ (No. 25); B: $\mathrm{C}_{10} \mathrm{H}_{12}$ (No. 33); C: $\mathrm{C}_{10} \mathrm{H}_{12}$ (No. 53); and D: $\mathrm{C}_{13} \mathrm{H}_{16}$ (No. 88).

Typical fragment ion spectra of detected partially saturated bicyclic and tricyclic compounds were showed in Figure S9. Methylstyrene is similar fragment ion spectra with 2,3-dihydro-1H-indene. However, due to its ethenyl, methylstyrene should be high active to undergo hydrogen transfer, cyclization and alkylation reactions. Thus No. 25 compound should be 2,3-dihydro- $1 H$-indene. It is easy to distinct methyl-2,3-dihydro-1H-indene (No. 33) and 1,2,3,4-tetrahydronaphthalene (No. 53). The methyl-2,3-dihydro- $1 H$-indene is easy to form ion with $\mathrm{m} / \mathrm{z}$ of 117 via losing the methyl, while 1,2,3,4-tetrahydronaphthalene tend to ring open, lose ethene and form ion with $\mathrm{m} / \mathrm{z}$ of 104 . No compound in NIST library matches the characteristic of fragment ion spectrum showed in Figure S9D. This compound is observed in samples from 1-butene and propene feeds. We supposed it to be 2,3,3a,4,5,6-hexahydro-1H-phenalene (No. 88, $\mathrm{C}_{13} \mathrm{H}_{16}$ ). With the fact that octahydropentalene, dihydroindene, and etc., tend to loss $\mathrm{C}_{2} \mathrm{H}_{4}$ group to form secondary ions, possible fragment mechanism of this compound is showed in Scheme S1. 
<smiles>CC1CCc2cccc3c2C1CCC3</smiles><smiles>CCCCC1CCc2cccc3c2C1CCC3</smiles>

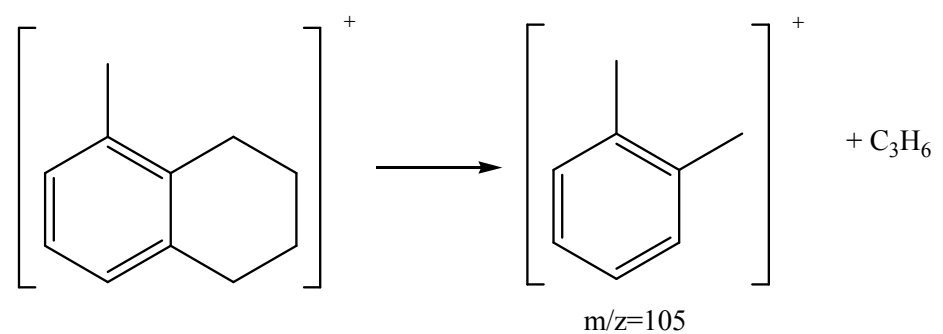

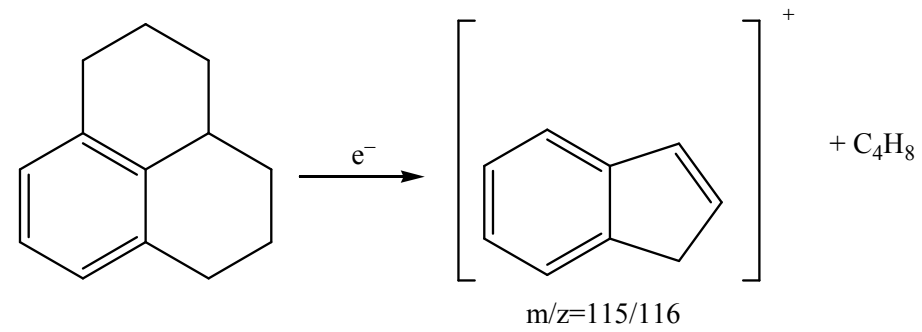

Scheme S1. Possible fragment mechanism of No. 88 compound. 

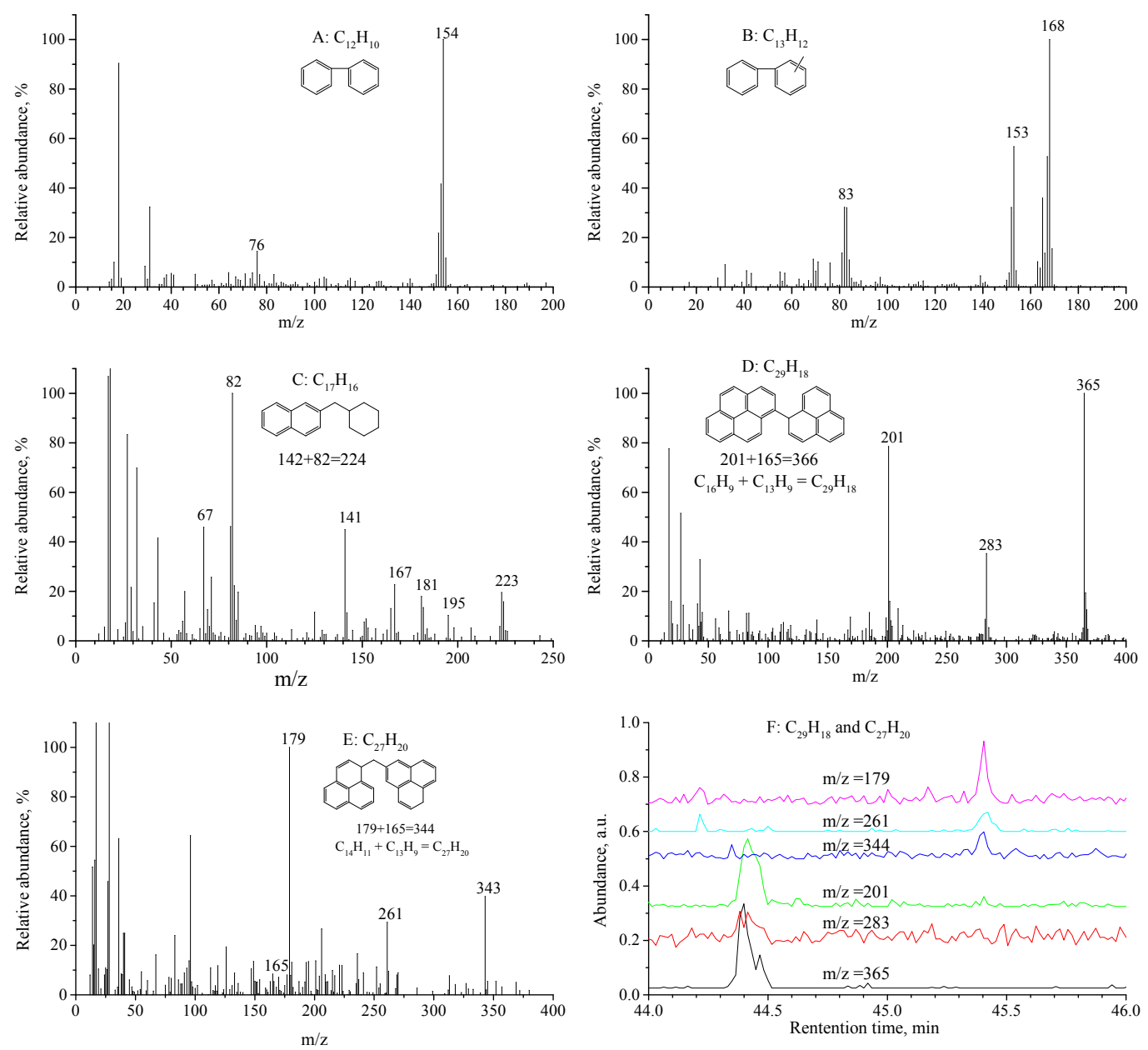

Figure S10. Typical fragment ion spectra of bridged compounds. A: $\mathrm{C}_{12} \mathrm{H}_{10}\left(\right.$ No. 78); B: $\mathrm{C}_{13} \mathrm{H}_{12}$ (No. 106); $\mathrm{C}: \mathrm{C}_{17} \mathrm{H}_{20}$ (No. 139); D: $\mathrm{C}_{29} \mathrm{H}_{18}$ (No. 140); $\mathrm{E}: \mathrm{C}_{27} \mathrm{H}_{20}$ (No. 141). F: Ion chromatogram of $\mathrm{C}_{29} \mathrm{H}_{18}$ and $\mathrm{C}_{27} \mathrm{H}_{20}$ (No. 140 and 141).

Typical bridged compounds were showed in Figure S10. Biphenyl (No. 78) and methylbiphenyl (No. 106) can well match with the ones in NIST MS database.

No standard mass spectrum is available for compounds No. 139, 140 and 141. The No. 139 compound is supposed to be cyclohexylmethylnaphthalene with fragment ions naphthalenylmethylium $(\mathrm{m} / \mathrm{z}=141)$ and cyclohexene $(\mathrm{m} / \mathrm{z}=82)$. The No. 140 should be phenalen-1-ylpyrene $(\mathrm{m} / \mathrm{z}=366)$ which bridged by pyrene $(\mathrm{m} / \mathrm{z}=202)$ and phenalene $(\mathrm{m} / \mathrm{z}=166)$. Possible fragment mechanism is showed in Scheme 2. 

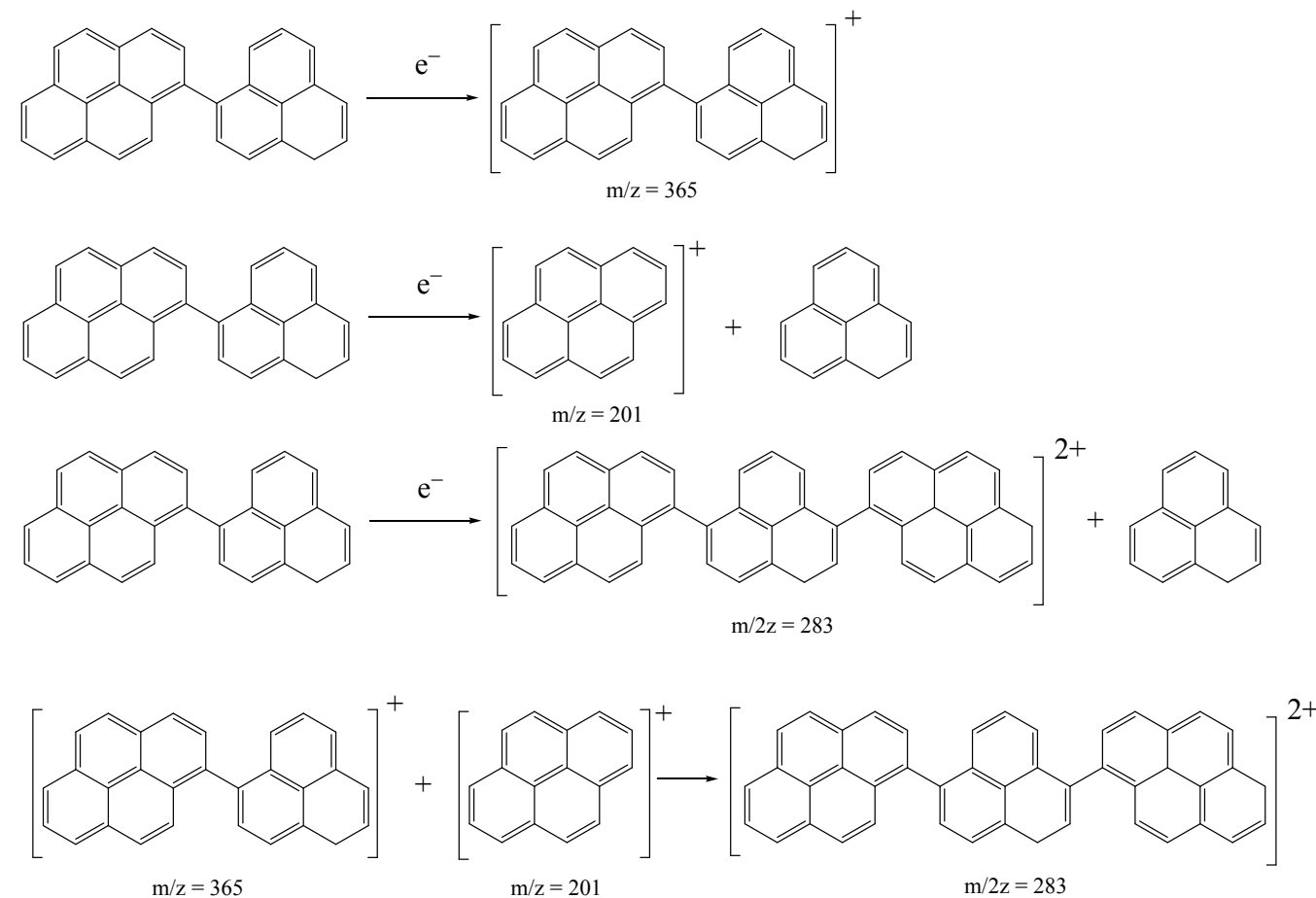

Scheme S2. Possible fragment mechanism of No. 140 compound.

Scheme S2 interpretates the formation of fragments at $\mathrm{m} / \mathrm{z}$ of 201,283 and 365. The intervals between 201, 283 and 365 are both 82, but the fragment ion at 82 was not observed. The reason should be that the fragment ion at 283 is a doubly charged ion formed by the addition of $\mathrm{C}_{16} \mathrm{H}_{9}{ }^{+}$and $\mathrm{C}_{29} \mathrm{H}_{18}{ }^{+}$. In fact, compounds with $\pi$ bond are easy to form doubly charged ion. ${ }^{3-5}$ The bridged polyaromatic structure should favor the delocalization of $\pi$ bond which stables of doubly charged ion and thus increases the intensity fragment at $\mathrm{m} / \mathrm{z}$ of 283 . However, the absence of fragment ions at 165 and 166 is still confusion.

The fragment mechanism of No. 141 (methylenebisphenalene) compound is similar to that of No. $\mathbf{1 4 0 .}$

\section{References}

1. Hu, B.; Mao, G.; Wang, D.; Fu, Y.; Wang, B.; Luo, M., Conversion and coking of olefins on SAPO-34. Catal. Sci. Technol. 2017, 7, (23), 5785-5794.

2. Luo, M.; Zang, H.; Hu, B.; Wang, B.; Mao, G., Evolution of confined species and their effects 
on catalyst deactivation and olefin selectivity in SAPO-34 catalyzed MTO process. RSC Adv. 2016, 6, (21), 17651-17658.

3. Mathur, B. P.; Burgess, E. M.; Bostwick, D. E.; Moran, T. F., Doubly charged ion mass spectra. 2-aromatic hydrocarbons. Org. Mass Spectrom. 1981, 16, (2), 92-98.

4. van der Burgt, P. J. M.; Dunne, M.; Gradziel, M. L., Hydrogen rearrangements in the fragmentation of anthracene by low-energy electron impact. The European Physical Journal D 2018, $72,(2), 31$.

5. Abliz, Z.; Aoki, J.; Ueda, T.; Kan, T.; Takekawa, M.; Iwashima, S., Electron impact mass spectra of polycyclic aromatic compounds with several types of pyridino- and benzobenzanthrone skeletons. Org. Mass Spectrom. 1993, 28, (5), 607-614. 